\title{
Asymptotic approximation of smooth convex bodies by general polytopes
}

\author{
Monika Ludwig
}

\section{Introduction and statement of results}

For the optimal approximation of convex bodies by inscribed or circumscribed polytopes there are precise asymptotic results with respect to different notions of distance. In this paper we want to derive some results on optimal approximation without restricting the polytopes to be inscribed or circumscribed.

Let $\mathcal{P}_{n}$ and $\mathcal{P}_{(n)}$ denote the set of polytopes with at most $n$ vertices and $n$ facets, respectively. For a convex body $C$, i.e., a compact convex set with non-empty interior, we are interested in the asymptotic behavior as $n \rightarrow \infty$ of

$$
\delta^{S}\left(C, \mathcal{P}_{n}\right)=\inf \left\{\delta^{S}(C, P): P \in \mathcal{P}_{n}\right\}
$$

and

$$
\delta^{S}\left(C, \mathcal{P}_{(n)}\right)=\inf \left\{\delta^{S}(C, P): P \in \mathcal{P}_{(n)}\right\}
$$

where $\delta^{S}(.,$.$) is the symmetric difference metric, i.e., \delta^{S}(C, D)=\operatorname{vol}(C \triangle D)$, the volume of the symmetric difference of $C$ and $D$.

Before giving our results, we will describe some of the known results in this area, for more information we refer to the survey [8]. Let $\mathcal{P}_{n}^{i}$ be the set of polytopes having at most $n$ vertices and being inscribed into $C$, i.e., their vertices are on the boundary of $C$, and let $\mathcal{P}_{(n)}^{c}$ be the set of polytopes having at most $n$ facets and being circumscribed to $C$, i.e., each facet touches $C$. Define $\delta^{S}\left(C, \mathcal{P}_{n}^{i}\right)$ and $\delta^{S}\left(C, \mathcal{P}_{(n)}^{c}\right)$ as above. For a convex body $C$ in Euclidean $d$-space $\mathbb{E}^{d}$ with boundary of differentiability class $\mathcal{C}^{2}$ and with positive Gaussian curvature $\kappa_{C}$, P.M. Gruber [9] proved that there are positive constants del $d_{d-1}$ and $\operatorname{div}_{d-1}$ (depending only on $d$ ) such that

$$
\delta^{S}\left(C, \mathcal{P}_{n}^{i}\right) \sim \frac{1}{2} \operatorname{del}_{d-1}\left(\int_{\mathrm{bd} C} \kappa_{C}(x)^{1 /(d+1)} d \sigma(x)\right)^{(d+1) /(d-1)} \frac{1}{n^{2 /(d-1)}}
$$

and

$$
\delta^{S}\left(C, \mathcal{P}_{(n)}^{c}\right) \sim \frac{1}{2} \operatorname{div}_{d-1}\left(\int_{\mathrm{bd} C} \kappa_{C}(x)^{1 /(d+1)} d \sigma(x)\right)^{(d+1) /(d-1)} \frac{1}{n^{2 /(d-1)}}
$$


as $n \rightarrow \infty$. Here $\sigma$ is the surface area measure in $\mathbb{E}^{d}$. For the case $d=2$ these results were stated by L. Fejes Tóth [4], p. 43, and proved by McClure and Vitale [11]. $\operatorname{del}_{d-1}$ and $\operatorname{div}_{d-1}$ are named after Delone triangulations and DirichletVorono $i$ tilings, because these are used in the proofs of the asymptotic formulae. Only the values $\operatorname{del}_{1}=1 / 6, \operatorname{del}_{2}=1 /(2 \sqrt{3}), \operatorname{div}_{1}=1 / 12$ and $\operatorname{div}_{2}=5 /(18 \sqrt{3})$ (see [6] and [7] for the determination of $\operatorname{del}_{2}$ and $\operatorname{div}_{2}$, respectively) are known explicitly.

For approximation without restricting the polytopes to be inscribed or circumscribed the following is known. Let $C$ be a convex body in $\mathbb{E}^{d}$ with boundary of differentiability class $\mathcal{C}^{2}$ and with positive Gaussian curvature $\kappa_{C}$. In the case $d=2$, L. Fejes Tóth [4], p. 43, stated the following asymptotic formula,

$$
\delta^{S}\left(C, \mathcal{P}_{n}\right)=\delta^{S}\left(C, \mathcal{P}_{(n)}\right) \sim \frac{1}{32}\left(\int_{\mathrm{bd} C} \kappa_{C}(x)^{1 / 3} d \sigma(x)\right)^{3} \frac{1}{n^{2}}
$$

as $n \rightarrow \infty$. For general $d$, Gruber and Kenderov [10] showed that there are positive constants $\alpha$ and $\beta$ (depending only on $d$ ) such that

$$
\frac{\alpha}{n^{2 /(d-1)}} \leq \delta^{S}\left(C, \mathcal{P}_{n}\right) \leq \frac{\beta}{n^{2 /(d-1)}}
$$

for $n=d+1, \ldots$. In our first theorem we give asymptotic results in the case of approximation by general polytopes.

Theorem 1 Let $C$ be a convex body in $\mathbb{E}^{d}$ with boundary of differentiability class $\mathcal{C}^{2}$ and with positive Gaussian curvature $\kappa_{C}$. Then there are positive constants $\operatorname{ldel}_{d-1}$ and $\operatorname{ldiv}_{d-1}$, depending only on $d$, such that

$$
\delta^{S}\left(C, \mathcal{P}_{n}\right) \sim \frac{1}{2} \operatorname{ldel}_{d-1}\left(\int_{\mathrm{bd} C} \kappa_{C}(x)^{1 /(d+1)} d \sigma(x)\right)^{(d+1) /(d-1)} \frac{1}{n^{2 /(d-1)}}
$$

and

$$
\delta^{S}\left(C, \mathcal{P}_{(n)}\right) \sim \frac{1}{2} \operatorname{ldiv}_{d-1}\left(\int_{\mathrm{bd} C} \kappa_{C}(x)^{1 /(d+1)} d \sigma(x)\right)^{(d+1) /(d-1)} \frac{1}{n^{2 /(d-1)}}
$$

as $n \rightarrow \infty$.

The constants $\operatorname{ldel}_{d-1}$ and $\operatorname{ldiv}_{d-1}$ are named after Laguerre, Delone, Dirichlet and Voronoi, because, instead of Voronoi tilings as in the case of circumscribed polytopes, Laguerre(-Delone-Dirichlet-Voronoi) tilings are used. It is easy to see that $\operatorname{ldel}_{1}=\operatorname{ldiv}_{1}=1 / 16$ which proves (1.1). For the case $d=3$, formula (1.3) was conjectured to hold in $[6]$ with the constant $\operatorname{ldel}_{2}=1 /(6 \sqrt{3})-1 /(8 \pi)$. In a joint paper with K. Böröczky, Jr. [3] it is shown that this is the correct value and that $\operatorname{ldiv}_{2}=5 /(18 \sqrt{3})-1 /(4 \pi)$. For $d>3$ it is probably difficult to determine the explicit values of $\operatorname{ldel}_{d-1}$ and $\operatorname{ldiv}_{d-1}$. 
$\int_{\mathrm{bd} C} \kappa_{C}(x)^{1 /(d+1)} d \sigma(x)$ is called the affine surface area of $C$. The affine isoperimetric inequality (cf. [12], p. 419) states that among all convex bodies of given volume the affine surface area is maximal for ellipsoids. Thus (1.3) and (1.4) imply that among all convex bodies of given volume ellipsoids are asymptotically worst approximated by polytopes.

As a second notion of distance we use the $L_{1}$-distance of the support functions of the convex bodies, i.e.,

$$
\delta_{1}(C, D)=\int_{S^{d-1}}\left|h_{C}(u)-h_{D}(u)\right| d \sigma(u)
$$

where $h_{C}(u)$ is the support function of $C$ (For notions of convex geometry not explained here, cf. [12].). For a convex body $C$ in $\mathbb{E}^{d}$ with boundary of differentiability class $\mathcal{C}^{2}$ and with positive Gaussian curvature $\kappa_{C}$, Glasauer and Gruber [5] proved that

$$
\delta_{1}\left(C, \mathcal{P}_{n}^{i}\right) \sim \frac{1}{2} \operatorname{div}_{d-1}\left(\int_{\mathrm{bd} C} \kappa_{C}(x)^{d /(d+1)} d \sigma(x)\right)^{(d+1) /(d-1)} \frac{1}{n^{2 /(d-1)}}
$$

and

$$
\delta_{1}\left(C, \mathcal{P}_{(n)}^{c}\right) \sim \frac{1}{2} \operatorname{del}_{d-1}\left(\int_{\mathrm{bd} C} \kappa_{C}(x)^{d /(d+1)} d \sigma(x)\right)^{(d+1) /(d-1)} \frac{1}{n^{2 /(d-1)}}
$$

as $n \rightarrow \infty$. As before, the case $d=2$ was treated by L. Fejes Tóth [4] and McClure and Vitale [11]. Our second theorem gives the respective results for approximation by general polytopes.

Theorem 2 Let $C$ be a convex body in $\mathbb{E}^{d}$ with boundary of differentiability class $\mathcal{C}^{2}$ and with positive Gaussian curvature $\kappa_{C}$. Then

$$
\delta_{1}\left(C, \mathcal{P}_{n}\right) \sim \frac{1}{2} \operatorname{ldiv}_{d-1}\left(\int_{\mathrm{bd} C} \kappa_{C}(x)^{d /(d+1)} d \sigma(x)\right)^{(d+1) /(d-1)} \frac{1}{n^{2 /(d-1)}}
$$

and

$$
\delta_{1}\left(C, \mathcal{P}_{(n)}\right) \sim \frac{1}{2} \operatorname{ldel}_{d-1}\left(\int_{\mathrm{bd} C} \kappa_{C}(x)^{d /(d+1)} d \sigma(x)\right)^{(d+1) /(d-1)} \frac{1}{n^{2 /(d-1)}}
$$

as $n \rightarrow \infty$.

Both, Theorem 1 and Theorem 2 can be obtained from the following more general result. Let $w: \mathbb{E}^{d} \rightarrow \mathbb{R}$ be a continuous and positive function and define

$$
\delta_{w}(C, D)=\int_{C \triangle D} w(x) d x
$$

where $d x=d x^{1} \ldots d x^{d}$ is the Lebesgue measure in $\mathbb{E}^{d}$. 
Theorem 3 Let $C$ be a convex body in $\mathbb{E}^{d}$ with boundary of differentiability class $\mathcal{C}^{2}$ and with positive Gaussian curvature $\kappa_{C}$. Then

$$
\delta_{w}\left(C, \mathcal{P}_{n}\right) \sim \frac{1}{2} \operatorname{ldel}_{d-1}\left(\int_{\mathrm{bd} C} w(x)^{(d-1) /(d+1)} \kappa_{C}(x)^{1 /(d+1)} d \sigma(x)\right)^{(d+1) /(d-1)} \frac{1}{n^{2 /(d-1)}}
$$

and

$$
\delta_{w}\left(C, \mathcal{P}_{(n)}\right) \sim \frac{1}{2} \operatorname{ldiv}_{d-1}\left(\int_{\mathrm{bd} C} w(x)^{(d-1) /(d+1)} \kappa_{C}(x)^{1 /(d+1)} d \sigma(x)\right)^{(d+1) /(d-1)} \frac{1}{n^{2 /(d-1)}}
$$

as $n \rightarrow \infty$.

Theorem 1 follows from this result by setting $w(x)=1$. To obtain Theorem 2 we follow an idea of Glasauer described in [5] and repeat some of the arguments given there. We may assume that the origin $o \in \operatorname{int} C$ (where int stands for interior) and set $w(x)=\|x\|^{-(d+1)}$ where $\|x\|$ is the Euclidean norm of $x$. Then, by using polar coordinates we have

$$
\begin{aligned}
\delta_{\|x\|^{-(d+1)}}\left(C^{*}, D^{*}\right) & =\int_{C^{*} \triangle D^{*}}\|x\|^{-(d+1)} d x=\int_{S^{d-1}}\left|\frac{1}{\rho_{C^{*}}(u)}-\frac{1}{\rho_{D^{*}}(u)}\right| d \sigma(u) \\
& =\int_{S^{d-1}}\left|h_{C}(u)-h_{D}(u)\right| d \sigma(u)=\delta_{1}(C, D)
\end{aligned}
$$

where $C^{*}=\left\{x \in \mathbb{E}^{d}:\langle x, y\rangle \leq 1\right.$ for all $\left.y \in C\right\}$ is the polar body of $C$ and $\rho_{C^{*}}(u)=1 / h_{C}(u)$ is the radial function of $C^{*}$. If $C$ is of class $\mathcal{C}^{2}$ and $\kappa_{C}>0$ then $C^{*}$ is of class $\mathcal{C}^{2}$ and $\kappa_{C^{*}}>0$ (see [12], p. 111). Therefore, it follows from Theorem 3 that

$$
\delta_{1}\left(C, \mathcal{P}_{n}\right) \sim \frac{1}{2} \operatorname{ldiv}_{d-1}\left(\int_{\mathrm{bd} C^{*}}\|x\|^{-(d-1)} \kappa_{C^{*}}(x)^{1 /(d+1)} d \sigma(x)\right)^{(d+1) /(d-1)} \frac{1}{n^{2 /(d-1)}}
$$

as $n \rightarrow \infty$. Since

$$
\int_{\mathrm{bd} C^{*}}\|x\|^{-(d-1)} \kappa_{C^{*}}(x)^{1 /(d+1)} d \sigma(x)=\int_{\mathrm{bd} C} \kappa_{C}(x)^{d /(d+1)} d \sigma(x)
$$

(see [5]), (1.5) is a consequence of (1.8) and similarly, (1.6) follows from (1.7). Thus, we only have to prove Theorem 3.

\section{Approximation of paraboloids}

As a first step in the proof of the asymptotic formulae (1.7) and (1.8) we consider the problem of approximating a paraboloid by a convex polyhedron and recall the connection of this question with Laguerre tilings. We denote by || volume in $\mathbb{E}^{d-1}$ and call balls and cubes in dimension $d-1$ circles and squares, respectively. 
Let $q(u)=u^{2}=\left(u^{1}\right)^{2}+\ldots+\left(u^{d-1}\right)^{2}$ where $u=\left(u^{1}, \ldots, u^{d-1}\right) \in \mathbb{E}^{d-1}$ and let $Q=\left\{\left(u, u^{d}\right): u \in \mathbb{E}^{d-1}, u^{d} \geq q(u)\right\}$. For a given compact and Jordan measurable set $J \subset \mathbb{E}^{d-1}$ we want to find a convex polyhedron $P$ with a given number of vertices or facets that minimizes $v_{P}(J)=\operatorname{vol}((P \triangle Q) \cap(J \times \mathbb{R}))$, i.e., the volume of that part of symmetric difference of $Q$ and $P$ which lies above $J$.

Let $P$ be convex polyhedron with facets $F_{1}, \ldots, F_{n} . P$ is the intersection of $n$ half-spaces which - in the cases we are interested in - can be described as $\left\{\left(u, u^{d}\right): u^{d} \geq l_{i}(u)\right\}$ where $l_{i}(u)=2 a_{i} u-a_{i}^{2}+s_{i}$ with $a_{i} \in \mathbb{E}^{d-1}$ and $s_{i} \in \mathbb{R}$, i.e., $\left(a_{i}, q\left(a_{i}\right)\right)$ is the point where the tangent plane to $Q$ is parallel to $F_{i}$. Let $l(u)=\max _{i=1, \ldots, n} l_{i}(u)$, then $P=\left\{\left(u, u^{d}\right): u^{d} \geq l(u)\right\}$ and

$$
v_{P}(J)=\int_{J}|q(u)-l(u)| d u .
$$

Set $L=\left\{\left(a_{1}, s_{1}\right), \ldots,\left(a_{n}, s_{n}\right)\right\} \cdot l(u)$ and $P$ are determined by $L$ and we will also write $v_{L}(J)$ for $v_{P}(J)$. We have

$$
\begin{aligned}
v_{L}(J) & =\int_{J}|q(u)-l(u)| d u \\
& =\int_{J}\left|u^{2}-\max _{i=1, \ldots, n} l_{i}(u)\right| d u \\
& =\int_{J}\left|\min \left\{(u-a)^{2}-s:(a, s) \in L\right\}\right| d u .
\end{aligned}
$$

Define $V_{i}=\left\{u \in J:\left(u-a_{i}\right)^{2}-s_{i} \leq\left(u-a_{j}\right)^{2}-s_{j}\right.$ for $\left.j=1, \ldots, n\right\}$. Then

$$
v_{L}(J)=\sum_{i=1}^{n} \int_{V_{i}}\left|\left(u-a_{i}\right)^{2}-s_{i}\right| d u .
$$

$V_{i}$ is the intersection of $J$ and the orthogonal projection of the facet $F_{i}$ of $P$ into $\mathbb{E}^{d-1}$. It is called Laguerre cell. The cells $V_{1}, \ldots, V_{n}$ form a tiling of $J$ which is called Laguerre tiling, Laguerre-Voronoi tiling, Dirichlet cell complex or power diagram of $L$ in $J$. In the case $s_{1}=\ldots=s_{n}$ we obtain (ordinary) DirichletVoronoi tilings. (For further information on Laguerre tilings, see, e.g., [1]).

First, we consider the problem of approximation by a polyhedron with a given number of facets. In this case it is easy to see that for a best approximating polyhedron every facet intersects $Q$ which implies $s_{i} \geq 0$ and we set $s_{i}=r_{i}^{2}$. Define

$$
\begin{aligned}
v_{(n)}(J) & =\inf \left\{v_{P}(J): P \in \mathcal{P}_{(n)}\right\} \\
& =\inf \left\{v_{L}(J): L=\left\{\left(a_{1}, r_{1}^{2}\right), \ldots,\left(a_{n}, r_{n}^{2}\right)\right\}\right\} .
\end{aligned}
$$

Since $v_{L}(J)$ depends continuously on $L$, this infimum is attained for some $L$. We have $v_{(n)}(J) \rightarrow 0$ as $n \rightarrow \infty$. Therefore, if $L_{n}$ are chosen such that $v_{L_{n}}(J)=$ $v_{(n)}(J)$, then as $n \rightarrow \infty$

$$
\max \left\{\operatorname{diam} V: V \text { is Laguerre cell of } L_{n}\right\} \rightarrow 0
$$


where diam stands for diameter. Further we will use that for $\mu>0$

$$
v_{(n)}(\mu J)=\mu^{d+1} v_{(n)}(J)
$$

which follows directly from the definition of $v_{(n)}(J)$.

We will now determine the asymptotic behavior of $v_{(n)}(J)$ as $n \rightarrow \infty$. The following lemma gives the definition of $\operatorname{ldiv}_{d-1}$. See Lemma 2 of [9] for the respective result for Dirichlet-Voronoi tilings.

Lemma 1 Let $I=\left\{u \in \mathbb{E}^{d-1}: 0 \leq u^{i} \leq 1\right\}$. For fixed $d$, there exists a positive constant $\operatorname{ldiv}_{d-1}$ such that

$$
v_{(n)}(I) \sim \frac{\operatorname{lidiv}_{d-1}}{n^{2 /(d-1)}}
$$

as $n \rightarrow \infty$.

Proof. For an $L=\left\{\left(a_{1}, r_{1}^{2}\right), \ldots,\left(a_{n}, r_{n}^{2}\right)\right\}$, denote by

$$
B_{i}=\left\{u \in \mathbb{E}^{d-1}:\left(u-a_{i}\right)^{2} \leq r_{i}^{2}\right\}
$$

the $i$-th Laguerre circle and by $V_{i}$ the respective Laguerre cell. Define

$$
v_{(n)}^{\prime}(I)=\inf \left\{v_{L}(I): L=\left\{\left(a_{1}, r_{1}^{2}\right), \ldots,\left(a_{n}, r_{n}^{2}\right)\right\} \text { with } B_{i} \subset I \text { for } i=1, \ldots, n\right\} .
$$

1. First, we show that there is a constant $\beta$ such that

$$
v_{(n)}^{\prime}(I) \leq \frac{\beta}{n^{2 /(d-1)}}
$$

for $n=1,2, \ldots$

Let $k=1,2, \ldots$ and take the common tiling of $I$ by $k^{d-1}$ squares of side-length $1 / k$. Let $a_{i}, i=1, \ldots, k^{d-1}$, be the centers of these squares, set $r_{1}=\ldots=r_{k^{d-1}}=$ 0 and $L=\left\{\left(a_{1}, r_{1}\right), \ldots,\left(a_{k^{d-1}}, r_{k^{d-1}}\right)\right\}$. Then

$v_{\left(k^{d-1}\right)}^{\prime}(I) \leq v_{L}(I) \leq k^{d-1} \int_{-\frac{1}{2 k}}^{\frac{1}{2 k}} \ldots \int_{-\frac{1}{2 k}}^{\frac{1}{2 k}}\left(\left(u^{1}\right)^{2}+\ldots+\left(u^{d-1}\right)^{2}\right) d u^{1} \ldots d u^{d-1}=\frac{d-1}{12 k^{2}}$.

For given $n$, choose $k$ such that $k^{d-1} \leq n \leq(k+1)^{d-1}$. Then, there is a $\beta$ such that

$$
v_{(n)}^{\prime}(I) n^{2 /(d-1)} \leq v_{\left(k^{d-1}\right)}^{\prime}(I)(k+1)^{2} \leq \frac{d-1}{12 k^{2}}(k+1)^{2} \leq \beta
$$

and (2.4) is proved.

2. The next step in our proof is to show that there is a positive constant $\alpha$ such that

$$
v_{(n)}^{\prime}(I) \geq \frac{\alpha}{n^{2 /(d-1)}}
$$

for $n=1,2, \ldots$ 
To show this we use the following mean inequality

$$
\sum_{i=1}^{n} \sigma_{i}^{(d+1) /(d-1)} n^{2 /(d-1)} \geq\left(\sum_{i=1}^{n} \sigma_{i}\right)^{(d+1) /(d-1)}
$$

where $\sigma_{i} \geq 0, i=1, \ldots, n$, and the following inequality for the polar moment of inertia for a measurable set $D \subset \mathbb{E}^{d-1}$ (see, e.g., [2], p. 51)

$$
\int_{D} u^{2} d u \geq \int_{B} u^{2} d u=\frac{d-1}{d+1} \kappa_{d-1}\left(\frac{|D|}{\kappa_{d-1}}\right)^{(d+1) /(d-1)}
$$

where $B$ is the $(d-1)$-dimensional circle of volume $|D|$ centered at the origin and $\kappa_{d-1}$ is the volume of the $(d-1)$-dimensional unit circle.

Let $L$ be chosen such that $v_{L}(I)=v_{(n)}^{\prime}(I)$ and let $l(u)$ be the piecewise linear function given by $L$. Then, as $\varepsilon \rightarrow 0$

$$
\int_{I}|q(u)-(l(u)+\varepsilon)| d u=v_{L}(I)-\varepsilon \sum_{i=1}^{n}\left|V_{i} \backslash B_{i}\right|+\varepsilon \sum_{i=1}^{n}\left|B_{i} \cap V_{i}\right|+o(\varepsilon) .
$$

Since $L$ is optimal, this implies

$$
\sum_{i=1}^{n}\left|B_{i} \cap V_{i}\right|=\sum_{i=1}^{n}\left|V_{i} \backslash B_{i}\right|
$$

For every facet $F_{i}$ of the polyhedron given by $L$, the cone with base $F_{i} \cap Q$ and apex at the point where the tangent plane of $Q$ is parallel to $F_{i}$ is contained in $Q \backslash P$ and has volume $r_{i}^{2}\left|B_{i} \cap V_{i}\right| / d$. Moreover, these cones have pairwise disjoint interiors. Thus,

$$
\sum_{i=1}^{n} r_{i}^{2}\left|B_{i} \cap V_{i}\right| \leq d \sum_{i=1}^{n} \int_{B_{i} \cap V_{i}}\left(r_{i}^{2}-\left(u-a_{i}\right)^{2}\right) d u .
$$

Consequently,

$$
\begin{aligned}
\sum_{i=1}^{n} \int_{B_{i} \cap V_{i}}\left(r_{i}^{2}-\left(u-a_{i}\right)^{2}\right) d u & =\sum_{i=1}^{n} r_{i}^{2}\left|B_{i} \cap V_{i}\right|-\sum_{i=1}^{n} \int_{B_{i} \cap V_{i}}\left(u-a_{i}\right)^{2} d u \\
& \leq d \sum_{i=1}^{n} \int_{B_{i} \cap V_{i}}\left(r_{i}^{2}-\left(u-a_{i}\right)^{2}\right) d u-\sum_{i=1}^{n} \int_{B_{i} \cap V_{i}}\left(u-a_{i}\right)^{2} d u
\end{aligned}
$$

and by (2.7)

$$
\begin{aligned}
(d-1) \sum_{i=1}^{n} \int_{B_{i} \cap V_{i}}\left(r_{i}^{2}-\left(u-a_{i}\right)^{2}\right) d u & \geq \sum_{i=1}^{n} \int_{B_{i} \cap V_{i}}\left(u-a_{i}\right)^{2} d u \\
& \geq \frac{d-1}{d+1} \kappa_{d-1} \sum_{i=1}^{n}\left(\frac{\left|B_{i} \cap V_{i}\right|}{\kappa_{d-1}}\right)^{(d+1) /(d-1)} .
\end{aligned}
$$


Therefore, by (2.6) and (2.8)

$$
\begin{aligned}
v_{(n)}^{\prime}(I) n^{2 /(d-1)} & \geq \sum_{i=1}^{n} \int_{B_{i} \cap V_{i}}\left(r_{i}^{2}-\left(u-a_{i}\right)^{2}\right) d u n^{2 /(d-1)} \\
& \geq \frac{1}{(d+1) \kappa_{d-1}^{2 /(d-1)}} \sum_{i=1}^{n}\left|B_{i} \cap V_{i}\right|^{(d+1) /(d-1)} n^{2 /(d-1)} \\
& \geq \frac{1}{(d+1) \kappa_{d-1}^{2 /(d-1)}}\left(\sum_{i=1}^{n}\left|B_{i} \cap V_{i}\right|\right)^{(d+1) /(d-1)} \\
& \geq \frac{1}{(d+1) \kappa_{d-1}^{2 /(d-1)}}\left(\frac{1}{2}\right)^{(d+1) /(d-1)}=\alpha
\end{aligned}
$$

and (2.5) is proved.

3. Define

$$
\operatorname{ldiv}_{d-1}=\liminf _{n \rightarrow \infty} v_{(n)}^{\prime}(I) n^{2 /(d-1)} .
$$

By $(2.5)$ and (2.4) $0<\operatorname{ldiv}_{d-1}<\infty$. We have to show that

$$
\operatorname{liv}_{d-1}=\lim _{n \rightarrow \infty} v_{(n)}^{\prime}(I) n^{2 /(d-1)} .
$$

To prove this it suffices to show the following for every $\lambda>1$. If $n_{0}$ is chosen such that

$$
v_{\left(n_{0}\right)}^{\prime}(I) n_{0}^{2 /(d-1)} \leq \lambda \operatorname{ldiv}_{d-1}
$$

then

$$
v_{(n)}^{\prime}(I) n^{2 /(d-1)} \leq \lambda^{2} \operatorname{ldiv}_{d-1}
$$

for all $n$ sufficiently large.

Let $k=1,2, \ldots$ and take the common tiling of $I$ by squares $I_{1}, \ldots, I_{k^{d-1}}$ of side-length $1 / k$. Choose $L_{j}$ such that $v_{\left(n_{0}\right)}^{\prime}\left(I_{j}\right)=v_{L_{j}}\left(I_{j}\right)$. Then, since in the definition of $v_{\left(n_{0}\right)}^{\prime}\left(I_{j}\right)$ we have $B=\left\{u \in \mathbb{E}^{d-1}:(u-a)^{2} \leq r^{2}\right\} \subset I_{j}$ for every $\left(a, r^{2}\right) \in L_{j}$, we obtain

$$
\int_{I_{j}}\left|\min \left\{(u-a)^{2}-r^{2}:\left(a, r^{2}\right) \in L_{1} \cup \ldots \cup L_{k^{d-1}}\right\}\right| d u \leq v_{L_{j}}\left(I_{j}\right),
$$

and by (2.3)

$$
v_{L_{j}}\left(I_{j}\right)=\frac{1}{k^{d+1}} v_{\left(n_{0}\right)}^{\prime}(I)
$$

for $j=1, \ldots, k^{d-1}$. Therefore,

$$
\begin{aligned}
v_{\left(n_{0} k^{d-1}\right)}^{\prime}(I) & \leq v_{L_{1} \cup \ldots \cup L_{k^{d-1}}}\left(I_{1} \cup \ldots \cup I_{k^{d-1}}\right) \\
& \leq v_{L_{1}}\left(I_{1}\right)+\ldots+v_{L_{k^{d-1}}}\left(I_{k^{d-1}}\right) \\
& \leq \frac{1}{k^{2}} v_{\left(n_{0}\right)}^{\prime}(I)
\end{aligned}
$$


and

$$
v_{\left(n_{0} k^{d-1}\right)}^{\prime}(I)\left(n_{0} k^{d-1}\right)^{2 /(d-1)} \leq v_{\left(n_{0}\right)}^{\prime}(I) n_{0}^{2 /(d-1)} .
$$

Choose $k_{0}$ such that $((k+1) / k)^{2} \leq \lambda$ for $k=k_{0}, k_{0}+1, \ldots$ For $n$ sufficiently large we can find a $k \geq k_{0}$ such that $n_{0} k^{d-1} \leq n \leq n_{0}(k+1)^{d-1}$. By (2.13) and (2.11) we have

$$
\begin{aligned}
v_{(n)}^{\prime}(I) n^{2 /(d-1)} & \leq v_{\left(n_{0} k^{d-1}\right)}^{\prime}(I)\left(n_{0}(k+1)^{d-1}\right)^{2 /(d-1)} \\
& \leq v_{\left(n_{0}\right)}^{\prime}(I) n_{0}^{2 /(d-1)}\left(\frac{k+1}{k}\right)^{2} \leq \lambda^{2} \operatorname{ldiv}_{d-1} .
\end{aligned}
$$

Thus, (2.12) and (2.10) are proved.

4. Obviously,

$$
v_{(n)}(I) \leq v_{(n)}^{\prime}(I)
$$

We have to show that

$$
\lim _{n \rightarrow \infty} v_{(n)}^{\prime}(I) n^{2 /(d-1)} \leq \liminf _{n \rightarrow \infty} v_{(n)}(I) n^{2 /(d-1)} .
$$

Let $\lambda>1$ be chosen and let $I^{\prime}$ be a square concentric with $I$ and with $\left|I^{\prime}\right|=1 / \lambda$. Choose $L_{n}$ such that $v_{(n)}(I)=v_{L_{n}}(I)$. (2.2) implies that for $n$ sufficiently large

$$
\bigcup\left\{V: V \text { is Laguerre cell of } L_{n} \text { with Laguerre circle } B \subset I\right\} \supset I^{\prime}
$$

and we denote by $L_{n}^{\prime}$ the subset of $L_{n}$ defining such cells. It follows from (2.3) and (2.4) that we can choose $n^{\prime} \leq(\lambda-1) n$ points $a_{1}, \ldots, a_{n^{\prime}}$ lying in $I \backslash I^{\prime}$ and define $L=\left\{\left(a_{1}, 0\right), \ldots,\left(a_{n^{\prime}}, 0\right)\right\}$ such that

$$
v_{L}\left(I \backslash I^{\prime}\right) \leq\left(1-\frac{1}{\lambda}\right)^{d+1} \frac{\beta}{((\lambda-1) n)^{2 /(d-1)}} .
$$

Combining this with (2.16) gives

$$
v_{L_{n}^{\prime} \cup L}(I) \leq v_{(n)}(I)+\left(1-\frac{1}{\lambda}\right)^{d+1} \frac{\beta}{((\lambda-1) n)^{2 /(d-1)}} .
$$

$L_{n}^{\prime} \cup L$ has less than $\lambda n$ cells and for all of its Laguerre circles $B$ we have $B \subset I$. Therefore,

$$
v_{(\lceil\lambda n\rceil)}^{\prime}(I) \leq v_{L_{n}^{\prime} \cup L}(I)
$$

and

$$
\begin{aligned}
\lim _{n \rightarrow \infty} v_{(\lceil\lambda n\rceil)}^{\prime}(I)(\lceil\lambda n\rceil)^{2 /(d-1)} & =\lim _{n \rightarrow \infty} v_{(\lceil\lambda n\rceil)}^{\prime}(I)(\lambda n)^{2 /(d-1)} \\
& \leq \lambda^{2 /(d-1)} \liminf _{n \rightarrow \infty} v_{(n)}(I) n^{2 /(d-1)}+\left(\frac{\lambda-1}{\lambda}\right)^{\left(d^{2}-3\right) /(d-1)} \beta .
\end{aligned}
$$


Since $\lambda>1$ was arbitrary, this shows (2.15). Combined with (2.10) this completes the proof of the lemma.

Next, we extend Lemma 1 from the unit square to Jordan measurable sets and from $q(u)=u^{2}$ to general positive definite quadratic forms. For a convex function $f: \mathbb{E}^{d-1} \rightarrow \mathbb{R}$ define

$$
v_{P}(J ; f)=\int_{J}|f(u)-l(u)| d u
$$

where $l(u)$ describes the convex polyhedron $P$, and define $v_{(n)}(J ; f)$ as above.

Lemma 2 Let $J \subset \mathbb{E}^{d-1}$ be compact and Jordan measurable and $q$ a positive definite quadratic form. Then

$$
v_{(n)}(J ; q) \sim \operatorname{lidiv}_{d-1}(\operatorname{det} q)^{1 /(d-1)}|J|^{(d+1) /(d-1)} \frac{1}{n^{2 /(d-1)}}
$$

as $n \rightarrow \infty$, where det stands for determinant.

Proof. We only have to prove the lemma for $q(u)=u^{2}$, the more general case can then be obtained by applying a linear transformation.

First, we show that

$$
\limsup _{n \rightarrow \infty} v_{(n)}(J) n^{2 /(d-1)} \leq \operatorname{ldiv}_{d-1}|J|^{(d+1) /(d-1)} .
$$

Let $\lambda>1$ be chosen. Since $J$ is Jordan measurable, we can find $m$ squares $I_{1}, \ldots, I_{m}$ of equal area $|I|$ such that

$$
J \subset \bigcup_{l=1}^{m} I_{l}
$$

and

$$
m|I| \leq \lambda|J|
$$

Thus, we have by (2.18), (2.10), (2.3), and (2.19)

$$
\begin{aligned}
v_{(k m)}(J)(k m)^{2 /(d-1)} & \leq \sum_{l=1}^{m} v_{(k)}^{\prime}\left(I_{l}\right) k^{2 /(d-1)} m^{2 /(d-1)} \\
& \leq \lambda \operatorname{lid}_{d-1}|I|^{(d+1) /(d-1)} m^{(d+1) /(d-1)} \\
& \leq \lambda^{(2 d) /(d-1)} \operatorname{div}_{d-1}|J|^{(d+1) /(d-1)}
\end{aligned}
$$

for $k$ sufficiently large. Choose $k_{0}$ such that for $k=k_{0}, k_{0}+1, \ldots(2.20)$ holds and $((k+1) / k)^{2 /(d-1)} \leq \lambda$. For sufficiently large $n$ we can find a $k \geq k_{0}$ such that $m k \leq n \leq m(k+1)$. Therefore,

$$
\begin{aligned}
v_{(n)}(J) n^{2 /(d-1)} & \leq v_{(k m)}(J)((k+1) m)^{2 /(d-1)} \\
& \leq \lambda^{(2 d) /(d-1)} \operatorname{liv}_{d-1}|J|^{(d+1) /(d-1)}\left(\frac{k+1}{k}\right)^{2 /(d-1)} \\
& \leq \lambda^{(3 d-1) /(d-1)} \operatorname{liv}_{d-1}|J|^{(d+1) /(d-1)}
\end{aligned}
$$

and (2.17) is proved. 
Second, we show that

$$
\liminf _{n \rightarrow \infty} v_{(n)}(J) n^{2 /(d-1)} \geq \lim _{d-1}|J|^{(d+1) /(d-1)} .
$$

Let $\lambda>1$ be chosen. Since $J$ is Jordan measurable, there are pairwise disjoint squares $I_{1}, \ldots, I_{m}$ such that

$$
\bigcup_{l=1}^{m} I_{l} \subset J
$$

and

$$
|J| \leq \lambda \sum_{l=1}^{m}\left|I_{l}\right|
$$

Choose $L$ such that $v_{(n)}(J)=v_{L}(J)$ and denote by $n_{l}$ the number of Laguerre cells of $L$ which intersect $I_{l}$. Because of (2.2), we see that no Laguerre cell of $L$ intersects two different squares for $n$ sufficiently large. Therefore,

$$
n_{1}+\ldots+n_{m} \leq n
$$

By Lemma 1 and (2.3) there is a $n_{0}$ such that

$$
v_{(k)}\left(I_{l}\right) \geq \frac{\operatorname{lidiv}_{d-1}}{\lambda}\left|I_{l}\right|^{(d+1) /(d-1)} \frac{1}{k^{2 /(d-1)}}
$$

for $k \geq n_{0}$ and $l=1, \ldots, m$. Since $v_{(n)}(J) \rightarrow 0$ as $n \rightarrow \infty$,

$$
n_{l} \geq n_{0} \quad \text { for } l=1, \ldots, m
$$

and $n$ sufficiently large. Therefore, we have by (2.22) and (2.25)

$$
v_{(n)}(J) \geq \sum_{l=1}^{m} v_{\left(n_{l}\right)}\left(I_{l}\right) \geq \frac{\operatorname{lidiv}_{d-1}}{\lambda} \sum_{l=1}^{m} \frac{\left|I_{l}\right|^{(d+1) /(d-1)}}{n_{l}^{2 /(d-1)}} .
$$

By using Hölder's inequality

$$
\sum_{l=1}^{m}\left|I_{l}\right|=\sum_{l=1}^{m}\left(\frac{\left|I_{l}\right|}{n_{l}^{2 /(d+1)}}\right) n_{l}^{2 /(d+1)} \leq\left(\sum_{l=1}^{m} \frac{\left|I_{l}\right|^{(d+1) /(d-1)}}{n_{l}^{2 /(d-1)}}\right)^{(d-1) /(d+1)}\left(\sum_{l=1}^{m} n_{l}\right)^{2 /(d+1)},
$$

(2.23) and (2.24), we obtain

$$
\begin{aligned}
v_{(n)}(J) & \geq \frac{\operatorname{lidiv}_{d-1}}{\lambda}\left(\sum_{l=1}^{m}\left|I_{l}\right|\right)^{(d+1) /(d-1)}\left(\frac{1}{\sum_{l=1}^{m} n_{l}}\right)^{2 /(d-1)} \\
& \geq \frac{\operatorname{ldiv}_{d-1}}{\lambda^{(2 d) /(d-1)}}|J|^{(d+1) /(d-1)} \frac{1}{n^{2 /(d-1)}}
\end{aligned}
$$

for $n$ sufficiently large and (2.21) is proved. 
As second case, we consider the problem of approximating a paraboloid by a convex polyhedron with $n$ vertices. Define

$$
\begin{aligned}
v_{n}(J) & =\inf \left\{v_{P}(J): P \in \mathcal{P}_{n}\right\} \\
& =\inf \left\{v_{L}(I): L \text { defines a Laguerre tiling of } I \text { with at most } n \text { vertices }\right\}
\end{aligned}
$$

and define $v_{n}(J ; f)$ as above. Similar to the case of approximation by polyhedra with $n$ facets we have the following results.

Lemma 3 Let $I=\left\{u \in \mathbb{E}^{d-1}: 0 \leq u^{i} \leq 1\right\}$. Then, for fixed $d$, there exists a positive constant ldel $_{d-1}$ such that

$$
v_{n}(I) \sim \frac{\operatorname{ldel}_{d-1}}{n^{2 /(d-1)}}
$$

as $n \rightarrow \infty$.

Lemma 4 Let $J \subset \mathbb{E}^{d-1}$ be compact and Jordan measurable and $q$ a positive definite quadratic form. Then

$$
v_{n}(J ; q) \sim \operatorname{ldel}_{d-1}(\operatorname{det} q)^{1 /(d-1)}|J|^{(d+1) /(d-1)} \frac{1}{n^{2 /(d-1)}}
$$

as $n \rightarrow \infty$.

We omit the proofs of these lemmata because they are similar to the proofs of Lemma 1 and Lemma 2. The upper and lower bounds for $v_{n}(I)$ needed in the proof of Lemma 3 can be obtained with the help of (1.2).

\section{Proof of Theorem 3}

We will only give the proof of (1.8). (1.7) can be obtained along similar lines.

3.1 We may assume that the origin $o \in \operatorname{int} C$. We use the following local representation of bd $C$ (compare [9]): Given $p \in \operatorname{bd} C$, consider the ray $R$ through $p$ starting at $o$ and let $H$ be a hyperplane with $C \cap H=\emptyset$ which intersects $R$ orthogonally. Choose a Cartesian coordinate system in $H$. Together with the normal unit vector of $H$ which points to $C$ it forms a Cartesian coordinate system in $\mathbb{E}^{d}$. We write " $"$ " for the orthogonal projection of $\mathbb{E}^{d}$ onto $H=\mathbb{E}^{d-1}$. Next, represent the lower side of bd $C$ in the form $\left\{(u, f(u)): u \in C^{\prime}\right\}$. Then $f=f_{p}: C^{\prime} \rightarrow \mathbb{R}$ is a convex function and $f \mid \operatorname{int} C^{\prime}$ is of class $\mathcal{C}^{2}$. To each $u \in \operatorname{int} C^{\prime}$ we let correspond the quadratic form $q_{u}=q_{p, u}$ defined by

$$
q_{u}(s)=\sum_{i, j} f_{{ }_{i j}}(u) s^{i} s^{j} \text { for } s=\left(s^{1}, \ldots, s^{d-1}\right) \in H=\mathbb{E}^{d-1}
$$


$(i, j=1, \ldots, d-1)$. Here we denote by $f,{ }_{j}$ the first partial derivatives of $f$. If $x=(u, f(u)) \in \operatorname{bd} C$, we write also $\kappa_{C}(u)$ for $\kappa_{C}(x)$ and have

$$
\kappa_{C}(u)=\frac{\operatorname{det} q_{u}}{\left(1+\sum_{j} f,{ }_{j}(u)^{2}\right)^{(d+1) / 2}}
$$

for $u \in \operatorname{int} C^{\prime}$.

3.2 Choose $\lambda>1$. Since $\kappa_{C}>0$, the quadratic forms $q_{u}$ all are positive definite. Their coefficients are continuous. Therefore, we can choose an open convex neighbourhood $U^{\prime}$ of $p^{\prime}$ in $\operatorname{int} C^{\prime}$ such that

$$
\frac{1}{\lambda} q_{p^{\prime}}(s) \leq q_{u}(s) \leq \lambda q_{p^{\prime}}(s)
$$

for $u \in U^{\prime}, s \in \mathbb{E}^{d-1}$, and

$$
\frac{1}{\lambda} \operatorname{det} q_{p^{\prime}} \leq \operatorname{det} q_{u} \leq \lambda \operatorname{det} q_{p^{\prime}}
$$

for $u \in U^{\prime}$. We denote by $U$ the inverse image of $U^{\prime}$ with respect to the projection " " on the lower side of bd $C$.

The main step of the proof is to show the following result.

Proposition 1 Let $J$ be a compact Jordan measurable subset of $U^{\prime}$. Then

$$
\begin{aligned}
\frac{\operatorname{ldiv}_{d-1}}{2 \lambda^{9 d}} & \left(\operatorname{det} q_{p^{\prime}}\right)^{1 /(d-1)}|J|^{(d+1) /(d-1)} \frac{1}{k^{2 /(d-1)}} \\
& \leq v_{(k)}(J ; f) \leq \frac{\lambda^{9 d}}{2} \operatorname{ldiv}_{d-1}\left(\operatorname{det} q_{p^{\prime}}\right)^{1 /(d-1)}|J|^{(d+1) /(d-1)} \frac{1}{k^{2 /(d-1)}}
\end{aligned}
$$

for $k$ sufficiently large.

Proof. First, we establish the estimate from below for $v_{(k)}(J ; f)$. There is a piecewise linear convex function $l(u)=\max _{i=1, \ldots, k} l_{i}(u)$ such that

$$
v_{(k)}(J ; f)=\int_{J}|f(u)-l(u)| d u=\sum_{i=1}^{k} \int_{Q_{i}}\left|f(u)-l_{i}(u)\right| d u
$$

where $Q_{i}=\left\{u \in J: l_{i}(u) \geq l_{j}(u)\right.$ for $\left.j=1, \ldots, k\right\}$. We can choose $a_{i} \in J$ such that the tangent plane to $f(u)$ at $a_{i}$ is parallel to the plane determined by $l_{i}(u)$, i.e.,

$$
l_{i}(u)=\sum_{l=1}^{d-1} f_{, l}\left(a_{i}\right)\left(u^{l}-a_{i}^{l}\right)+l_{i}\left(a_{i}\right) .
$$

As in the case of paraboloids, it is easy to see that every $l_{i}(u)$ intersects $f(u)$, and we set $r_{i}^{2}=l\left(a_{i}\right)-f\left(a_{i}\right) \geq 0$. By Taylor's formula, there is, for fixed $u \in J$, a $\tau_{i}, 0<\tau_{i}<1$, such that

$$
\begin{aligned}
f(u)-l_{i}(u) & =f\left(a_{i}\right)+\sum_{l=1}^{d-1} f_{, l}\left(a_{i}\right)\left(u^{l}-a_{i}^{l}\right)+\frac{1}{2} q_{a_{i}+\tau_{i}\left(u-a_{i}\right)}\left(u-a_{i}\right)-l_{i}(u) \\
& =\frac{1}{2} q_{a_{i}+\tau_{i}\left(u-a_{i}\right)}\left(u-a_{i}\right)-r_{i}^{2}
\end{aligned}
$$


Define

$$
V_{i}=\left\{u \in J: \frac{1}{2 \lambda} q\left(u-a_{i}\right)-r_{i}^{2} \leq \frac{1}{2 \lambda} q\left(u-a_{j}\right)-r_{j}^{2} \text { for } j=1, \ldots, k\right\}
$$

and

$$
B_{i}=\left\{u \in \mathbb{E}^{d-1}: \frac{1}{2 \lambda} q\left(u-a_{i}\right) \leq r_{i}^{2}\right\}
$$

where we write $q$ for $q_{p^{\prime}}$, and set $B=\bigcup_{i=1}^{k} B_{i}$.

For $u \in J$ and $u \notin B$, we have by (3.3) and (3.2)

$$
f(u)-l_{i}(u)=\frac{1}{2} q_{a_{i}+\tau_{i}\left(u-a_{i}\right)}\left(u-a_{i}\right)-r_{i}^{2} \geq \frac{1}{2 \lambda} q\left(u-a_{i}\right)-r_{i}^{2} \geq 0 .
$$

Thus,

$$
\begin{aligned}
\int_{J \backslash B}|f(u)-l(u)| d u & =\sum_{i=1}^{k} \int_{Q_{i} \backslash B}\left(f(u)-l_{i}(u)\right) d u \\
& \geq \sum_{i=1}^{k} \int_{Q_{i} \backslash B}\left(\frac{1}{2 \lambda} q\left(u-a_{i}\right)-r_{i}^{2}\right) d u \\
& \geq \int_{J \backslash B}\left|\min \left\{\frac{1}{2 \lambda} q\left(u-a_{i}\right)-r_{i}^{2}: i=1, \ldots, k\right\}\right| d u .
\end{aligned}
$$

Next, we show that

$$
\int_{J \cap B}|f(u)-l(u)| d u \geq \frac{1}{\lambda^{8 d}} \int_{J \cap B}\left|\min \left\{\frac{1}{2 \lambda} q\left(u-a_{i}\right)-r_{i}^{2}: i=1, \ldots, k\right\}\right| d u .
$$

Note that by (3.2) and (3.3)

$$
\frac{1}{2 \lambda} q\left(u-a_{i}\right) \leq \frac{1}{2} q_{a_{i}+\tau_{i}\left(u-a_{i}\right)}\left(u-a_{i}\right) \leq\left|f(u)-l_{i}(u)\right|+r_{i}^{2} .
$$

We have

$$
\int_{J \cap B}|f(u)-l(u)| d u \geq \sum_{i=1}^{k} \int_{V_{i} \cap B_{i}}\left|f(u)-l_{i}(u)\right| d u-\sum_{i=1}^{k} \int_{V_{i} \cap B_{i}}\left|l(u)-l_{i}(u)\right| d u .
$$

We need estimates for the right-hand side of (3.7). For the first expression we have by (3.3), (3.2) and (3.6)

$$
\begin{aligned}
\left|f(u)-l_{i}(u)\right| & =\left|\frac{1}{2} q_{a_{i}+\tau_{i}\left(u-a_{i}\right)}\left(u-a_{i}\right)-r_{i}^{2}\right| \\
& \geq\left|\frac{1}{2 \lambda} q\left(u-a_{i}\right)-r_{i}^{2}\right|-\frac{1}{2}\left|q_{a_{i}+\tau_{i}\left(u-a_{i}\right)}\left(u-a_{i}\right)-\frac{1}{\lambda} q\left(u-a_{i}\right)\right| \\
& \geq\left|\frac{1}{2 \lambda} q\left(u-a_{i}\right)-r_{i}^{2}\right|-\frac{\lambda^{2}-1}{2 \lambda} q\left(u-a_{i}\right) \\
& \geq\left|\frac{1}{2 \lambda} q\left(u-a_{i}\right)-r_{i}^{2}\right|-\left(\lambda^{2}-1\right)\left|f(u)-l_{i}(u)\right|-\left(\lambda^{2}-1\right) r_{i}^{2}
\end{aligned}
$$


and

$$
\left|f(u)-l_{i}(u)\right| \geq \frac{1}{\lambda^{2}}\left|\frac{1}{2 \lambda} q\left(u-a_{i}\right)-r_{i}^{2}\right|-\frac{\lambda^{2}-1}{\lambda^{2}} r_{i}^{2} .
$$

Thus

$\sum_{i=1}^{k} \int_{V_{i} \cap B_{i}}\left|f(u)-l_{i}(u)\right| d u \geq \frac{1}{\lambda^{2}} \sum_{i=1}^{k} \int_{V_{i} \cap B_{i}}\left|\frac{1}{2 \lambda} q\left(u-a_{i}\right)-r_{i}^{2}\right| d u-\frac{\lambda^{2}-1}{\lambda^{2}} \sum_{i=1}^{k} r_{i}^{2}\left|V_{i} \cap B_{i}\right|$.

As in (2.9), we have

$$
\sum_{i=1}^{k} r_{i}^{2}\left|V_{i} \cap B_{i}\right| \leq d \sum_{i=1}^{k} \int_{V_{i} \cap B_{i}}\left|\frac{1}{2 \lambda} q\left(u-a_{i}\right)-r_{i}^{2}\right| d u .
$$

Therefore, we get for (3.8)

$$
\sum_{i=1}^{k} \int_{V_{i} \cap B_{i}}\left|f(u)-l_{i}(u)\right| d u \geq \frac{1-\left(\lambda^{2}-1\right) d}{\lambda^{2}} \sum_{i=1}^{k} \int_{V_{i} \cap B_{i}}\left|\frac{1}{2 \lambda} q\left(u-a_{i}\right)-r_{i}^{2}\right| d u .
$$

For the second expression on the right-hand side of (3.7) we obtain the following. For $u \in Q_{j} \cap V_{i}$, we have by (3.3)

$$
\begin{aligned}
0 \leq l(u)-l_{i}(u)= & l_{j}(u)-l_{i}(u) \\
= & \left(f(u)-l_{i}(u)\right)-\left(f(u)-l_{j}(u)\right) \\
= & \left(\frac{1}{2} q_{a_{i}+\tau_{i}\left(u-a_{i}\right)}\left(u-a_{i}\right)-r_{i}^{2}\right)-\left(\frac{1}{2} q_{a_{j}+\tau_{j}\left(u-a_{j}\right)}\left(u-a_{j}\right)-r_{j}^{2}\right) \\
= & \left(\frac{1}{2 \lambda} q\left(u-a_{i}\right)-r_{i}^{2}\right)-\left(\frac{1}{2 \lambda} q\left(u-a_{j}\right)-r_{j}^{2}\right) \\
& +\left(\frac{1}{2} q_{a_{i}+\tau_{i}\left(u-a_{i}\right)}\left(u-a_{i}\right)-\frac{1}{2 \lambda} q\left(u-a_{i}\right)\right) \\
& -\left(\frac{1}{2} q_{a_{j}+\tau_{j}\left(u-a_{j}\right)}\left(u-a_{j}\right)-\frac{1}{2 \lambda} q\left(u-a_{j}\right)\right) .
\end{aligned}
$$

By the definition of $V_{i}$, we have for $u \in V_{i}$

$$
\frac{1}{2 \lambda} q\left(u-a_{i}\right)-r_{i}^{2} \leq \frac{1}{2 \lambda} q\left(u-a_{j}\right)-r_{j}^{2}
$$

Thus, we obtain by $(3.2)$

$$
\begin{aligned}
l(u)-l_{i}(u) & \leq\left(\frac{1}{2} q_{a_{i}+\tau_{i}\left(u-a_{i}\right)}\left(u-a_{i}\right)-\frac{1}{2 \lambda} q\left(u-a_{i}\right)\right) \\
& \leq \frac{\lambda^{2}-1}{2 \lambda} q\left(u-a_{i}\right) \\
& \leq\left(\lambda^{2}-1\right)\left(\left|\frac{1}{2 \lambda} q\left(u-a_{i}\right)-r_{i}^{2}\right|+r_{i}^{2}\right)
\end{aligned}
$$


which holds for all $u \in V_{i}$. Using (3.9) now gives

$$
\begin{aligned}
\sum_{i=1}^{k} \int_{V_{i} \cap B_{i}} & \left|l(u)-l_{i}(u)\right| d u \\
\leq & \left(\lambda^{2}-1\right)\left(\sum_{i=1}^{k} \int_{V_{i} \cap B_{i}}\left|\frac{1}{2 \lambda} q\left(u-a_{i}\right)-r_{i}^{2}\right| d u+\sum_{i=1}^{k} r_{i}^{2}\left|V_{i} \cap B_{i}\right|\right) \\
\leq & \left(\lambda^{2}-1\right)(d+1) \sum_{i=1}^{k} \int_{V_{i} \cap B_{i}}\left|\frac{1}{2 \lambda} q\left(u-a_{i}\right)-r_{i}^{2}\right| d u
\end{aligned}
$$

Using this and (3.10), we obtain for (3.7)

$$
\int_{J \cap B}|f(u)-l(u)| d u \geq \frac{1}{\lambda^{8 d}} \sum_{i=1}^{k} \int_{V_{i} \cap B_{i}}\left|\frac{1}{2 \lambda} q\left(u-a_{i}\right)-r_{i}^{2}\right| d u
$$

for $\lambda$ not too large, which proves (3.5).

Combining (3.4) and (3.5) and using Lemma 2 gives

$$
\begin{aligned}
\int_{J}|f(u)-l(u)| d u & \geq \frac{1}{\lambda^{8 d}} v_{(k)}\left(J ; \frac{1}{2 \lambda} q\right) \\
& \geq \frac{1}{2 \lambda^{8 d+1}} \operatorname{ldiv}_{d-1}(\operatorname{det} q)^{1 /(d-1)}|J|^{(d+1) /(d-1)} \frac{1}{k^{2 /(d-1)}}
\end{aligned}
$$

for $k$ sufficiently large, i.e., the estimate from below is proved.

Second, we establish the estimate from above for $v_{(k)}(J ; f)$. Choose $a_{1}, \ldots$, $a_{k}$ and $r_{1}, \ldots, r_{k}$ such that

$$
v_{(k)}\left(J ; \frac{\lambda}{2} q\right)=\sum_{i=1}^{k} \int_{V_{i}}\left|\frac{\lambda}{2} q\left(u-a_{i}\right)-r_{i}^{2}\right| d u
$$

where $V_{i}=\left\{u \in J: \lambda / 2 q\left(u-a_{i}\right)-r_{i}^{2} \leq \lambda / 2 q\left(u-a_{j}\right)-r_{j}^{2}\right.$ for $\left.j=1, \ldots, k\right\}$. Define

$$
l_{i}(u)=f\left(a_{i}\right)+\sum_{l=1}^{d-1} f_{, l}\left(a_{i}\right)\left(u^{l}-a_{i}^{l}\right)+r_{i}^{2},
$$

$Q_{i}=\left\{u \in J: l_{i}(u) \geq l_{j}(u)\right.$ for $\left.j=1, \ldots, k\right\}$, and $D_{i}=\left\{u \in \mathbb{E}^{d-1}: f(u) \leq\right.$ $\left.l_{i}(u)\right\}$ for $i=1, \ldots, k$. Set $l(u)=\max _{i=1, \ldots, k} l_{i}(u)$ and $D=\bigcup_{i=1}^{k} D_{i}$.

For $u \notin D$, we have by (3.3) and (3.2)

$$
0 \leq f(u)-l_{i}(u) \leq \frac{\lambda}{2} q\left(u-a_{i}\right)-r_{i}^{2} .
$$

Thus, by the definition of $l(u)$ we have

$$
\sum_{i=1}^{k} \int_{V_{i} \backslash D}\left|\frac{\lambda}{2} q\left(u-a_{i}\right)-r_{i}^{2}\right| d u \geq \sum_{i=1}^{k} \int_{V_{i} \backslash D}\left(f(u)-l_{i}(u)\right) d u
$$




$$
\begin{aligned}
& \geq \sum_{i=1}^{k} \int_{V_{i} \backslash D}(f(u)-l(u)) d u \\
& =\int_{J \backslash D}|f(u)-l(u)| d u .
\end{aligned}
$$

Next, we show that

$$
\sum_{i=1}^{k} \int_{V_{i} \cap D}\left|\frac{\lambda}{2} q\left(u-a_{i}\right)-r_{i}^{2}\right| d u \geq \frac{1}{\lambda^{8 d}} \int_{J \cap D}|f(u)-l(u)| d u .
$$

Note that by the definition of the $V_{i}$ 's

$$
\sum_{i=1}^{k} \int_{V_{i} \cap D}\left|\frac{\lambda}{2} q\left(u-a_{i}\right)-r_{i}^{2}\right| d u=\int_{J \cap D}\left|\min \left\{\frac{\lambda}{2} q\left(u-a_{j}\right)-r_{j}^{2}: j=1, \ldots, k\right\}\right| d u .
$$

We have

$$
\begin{aligned}
& \left|\min \left\{\frac{\lambda}{2} q\left(u-a_{j}\right)-r_{j}^{2}: j=1, \ldots, k\right\}\right| \geq \\
& \left|\frac{\lambda}{2} q\left(u-a_{i}\right)-r_{i}^{2}\right|-\left|\left(\frac{\lambda}{2} q\left(u-a_{i}\right)-r_{i}^{2}\right)-\min \left\{\frac{\lambda}{2} q\left(u-a_{j}\right)-r_{j}^{2}: j=1, \ldots, k\right\}\right| .
\end{aligned}
$$

For the right-hand side of (3.13), we obtain the following. By (3.3) and (3.2),

$$
\begin{aligned}
\left|\frac{\lambda}{2} q\left(u-a_{i}\right)-r_{i}^{2}\right| & \geq\left|f(u)-l_{i}(u)\right|-\left|\frac{\lambda}{2} q\left(u-a_{i}\right)-\frac{1}{2} q_{a_{i}+\tau_{i}\left(u-a_{i}\right)}\left(u-a_{i}\right)\right| \\
& \geq\left|f(u)-l_{i}(u)\right|-\frac{\lambda^{2}-1}{2 \lambda} q\left(u-a_{i}\right) .
\end{aligned}
$$

For $u \in V_{j} \cap Q_{i}$, we see by the definition of $Q_{i}$ and (3.2)

$$
\begin{aligned}
\mid & \left(\frac{\lambda}{2} q\left(u-a_{i}\right)-r_{i}^{2}\right)-\min \left\{\frac{\lambda}{2} q\left(u-a_{j}\right)-r_{j}^{2}: j=1, \ldots, k\right\} \mid \\
= & \left(r_{j}^{2}-\frac{\lambda}{2} q\left(u-a_{j}\right)\right)-\left(r_{i}^{2}-\frac{\lambda}{2} q\left(u-a_{i}\right)\right) \\
= & \left(l_{j}(u)-f(u)\right)-\left(l_{i}(u)-f(u)\right)-\left(\frac{\lambda}{2} q\left(u-a_{j}\right)-\frac{1}{2} q_{a_{j}+\tau_{j}\left(u-a_{j}\right)}\left(u-a_{j}\right)\right) \\
& \quad+\left(\frac{\lambda}{2} q\left(u-a_{i}\right)-\frac{1}{2} q_{a_{i}+\tau_{i}\left(u-a_{i}\right)}\left(u-a_{i}\right)\right) \\
\leq & \frac{\lambda}{2} q\left(u-a_{i}\right)-\frac{1}{2} q_{a_{i}+\tau_{i}\left(u-a_{i}\right)}\left(u-a_{i}\right) \\
\leq & \frac{\lambda^{2}-1}{2 \lambda} q\left(u-a_{i}\right) \\
\leq & \left(\lambda^{2}-1\right)\left|f(u)-l_{i}(u)\right|+\left(\lambda^{2}-1\right) r_{i}^{2} .
\end{aligned}
$$

Combining this with (3.14) gives for (3.13)

$$
\begin{aligned}
\mid \min \left\{\frac{\lambda}{2} q\left(u-a_{j}\right)-r_{j}^{2}\right. & : j=1, \ldots, k\} \mid \\
\geq & \left(1-2\left(\lambda^{2}-1\right)\right)\left|f(u)-l_{i}(u)\right|-2\left(\lambda^{2}-1\right) r_{i}^{2} .
\end{aligned}
$$


Therefore,

$$
\begin{aligned}
\sum_{i=1}^{k} \int_{V_{i} \cap D}\left|\frac{\lambda}{2} q\left(u-a_{i}\right)-r_{i}^{2}\right| d u \geq & \left(1-2\left(\lambda^{2}-1\right)\right) \sum_{i=1}^{k} \int_{Q_{i} \cap D_{i}}\left|f(u)-l_{i}(u)\right| d u \\
& -2\left(\lambda^{2}-1\right) \sum_{i=1}^{k} r_{i}^{2}\left|Q_{i} \cap D_{i}\right| .
\end{aligned}
$$

Since we have as in (3.9)

$$
\sum_{i=1}^{k} r_{i}^{2}\left|Q_{i} \cap D_{i}\right| \leq d \sum_{i=1}^{k} \int_{Q_{i} \cap D_{i}}\left|f(u)-l_{i}(u)\right| d u,
$$

we obtain

$$
\sum_{i=1}^{k} \int_{V_{i} \cap D}\left|\frac{\lambda}{2} q\left(u-a_{i}\right)-r_{i}^{2}\right| d u \geq\left(1-2(d+1)\left(\lambda^{2}-1\right)\right) \int_{J \cap D}|f(u)-l(u)| d u
$$

and (3.12) follows. Combining (3.11) and (3.12) completes the proof of Proposition 1.

3.3 Further we use the following decomposition of $C$, see [9].

Proposition 2 There is a tiling of $\mathbb{E}^{d}$ with finitely many closed cones $C_{l}, l=$ $1, \ldots, m$, with common apex $o$, each of which has the following property: There is a point $p_{l} \in C_{l} \cap \mathrm{bd} C=T_{l}$ with corresponding $R_{l}, H_{l}, U_{l}, U_{l}^{\prime}, f_{l}=f_{p_{l}}, q_{u}=q_{p_{l}, u}$, $q_{l}=q_{p_{l}, p_{l}^{\prime}}$, and $w_{l}=w\left(p_{l}\right)$ such that

$$
\begin{gathered}
T_{l} \subset U_{l}, \\
\frac{1}{\lambda} q_{l}(s) \leq q_{u}(s) \leq \lambda q_{l}(s) \quad \text { for } u \in U_{l}^{\prime}, s \in \mathbb{E}^{d-1}, \\
\frac{1}{\lambda} \operatorname{det} q_{l} \leq \operatorname{det} q_{u} \leq \lambda \operatorname{det} q_{l} \quad \text { for } u \in U_{l}^{\prime},
\end{gathered}
$$

and

$$
\frac{1}{\lambda} w_{l} \leq w(x) \leq \lambda w_{l}
$$

for $x \in C_{l}$ and $x$ at a distance less than $\lambda-1$ from $T_{l}$. 
By our choice of $T_{l},(3.1),(3.15)$ and (3.16) we obtain the following.

$$
\begin{aligned}
& \int_{\operatorname{bd} C} w(x)^{(d-1) /(d+1)} \kappa_{C}(x)^{1 /(d+1)} d \sigma(x) \\
& \quad=\sum_{l=1}^{m} \int_{T_{l}^{\prime}} w((u, f(u)))^{(d-1) /(d+1)} \frac{\left(\operatorname{det} q_{u}\right)^{1 /(d+1)}}{\left(1+\sum_{k=1}^{d-1} f_{, k}(u)^{2}\right)^{1 / 2}}\left(1+\sum_{k=1}^{d-1} f_{, k}(u)^{2}\right)^{1 / 2} d u \\
& \left\{\begin{array}{l}
\leq \lambda \sum_{l=1}^{m} w_{l}^{(d-1) /(d+1)}\left(\operatorname{det} q_{l}\right)^{1 /(d+1)}\left|T_{l}^{\prime}\right| \\
\geq \frac{1}{\lambda} \sum_{l=1}^{m} w_{l}^{(d-1) /(d+1)}\left(\operatorname{det} q_{l}\right)^{1 /(d+1)}\left|T_{l}^{\prime}\right| .
\end{array}\right.
\end{aligned}
$$

3.4 Next, we give an estimate from below for $\delta_{w}\left(C, \mathcal{P}_{(n)}\right)$.

Proposition 3 For all $n$ sufficiently large,

$\delta_{w}\left(C, \mathcal{P}_{(n)}\right) \geq \frac{1}{2 \lambda^{12 d}} \operatorname{ldiv}_{d-1}\left(\int_{\mathrm{bd} C} w(x)^{(d-1) /(d+1)} \kappa_{C}(x)^{1 /(d+1)} d \sigma(x)\right)^{(d+1) /(d-1)} \frac{1}{n^{2 /(d-1)}}$.

Proof. There is a $P_{n} \in \mathcal{P}_{(n)}$ such that $\delta_{w}\left(C, P_{n}\right)=\delta_{w}\left(C, \mathcal{P}_{(n)}\right)$. Denote by $n_{l}$ the number of facets of $P_{n}$ which lie entirely in $C_{l}$. Then

$$
n_{1}+\ldots+n_{m} \leq n .
$$

Choose $n_{0}$ so large that for $k \geq n_{0}$ Proposition 1 holds for $f=f_{l}$ and $J=T_{l}^{\prime}$ for $l=1, \ldots, m . \kappa_{C}>0$ implies that $C$ is strictly convex. Since $P_{n} \stackrel{\delta_{w}}{\rightarrow} C$ as $n \rightarrow \infty$, this implies $\max \left\{\operatorname{diam} F: F\right.$ is a facet of $\left.P_{n}\right\} \rightarrow 0$ as $n \rightarrow \infty$. Therefore, we have

$$
n_{l} \geq n_{0}
$$

for $n$ sufficiently large. We see by (3.16), by our choice of $C_{l}$, the definition of $v_{(k)}\left(T_{l}^{\prime} ; f_{l}\right)$ and Proposition 1 that

$$
\begin{aligned}
\delta_{w}\left(C, P_{n}\right) & \geq \frac{1}{\lambda} \sum_{l=1}^{m} w_{l}\left(\text { volume of the subset of } C \triangle P_{n} \text { which lies in } C_{l}\right) \\
& \geq \frac{1}{\lambda^{2}} \sum_{l=1}^{m} w_{l} v_{\left(n_{l}\right)}\left(T_{l}^{\prime} ; f_{l}\right) \\
& \geq \frac{1}{2 \lambda^{9 d+2}} \operatorname{ldiv}_{d-1} \sum_{l=1}^{m} w_{l}\left(\operatorname{det} q_{l}\right)^{1 /(d-1)}\left|T_{l}^{\prime}\right|^{(d+1) /(d-1)} \frac{1}{n_{l}^{2 /(d-1)}} .
\end{aligned}
$$

By Hölder's inequality

$$
\begin{aligned}
& \sum_{l=1}^{m} w_{l}^{(d-1) /(d+1)}\left(\operatorname{det} q_{l}\right)^{1 /(d+1)}\left|T_{l}^{\prime}\right| \\
& \quad=\sum_{l=1}^{m}\left(w_{l}^{(d-1) /(d+1)}\left(\operatorname{det} q_{l}\right)^{1 /(d+1)}\left|T_{l}^{\prime}\right| \frac{1}{n_{l}^{2 /(d+1)}}\right) n_{l}^{2 /(d+1)} \\
& \quad \leq\left(\sum_{l=1}^{m} w_{l}\left(\operatorname{det} q_{l}\right)^{1 /(d-1)}\left|T_{l}^{\prime}\right|^{(d+1) /(d-1)} \frac{1}{n_{l}^{2 /(d-1)}}\right)^{(d-1) /(d+1)}\left(\sum_{l=1}^{m} n_{l}\right)^{2 /(d+1)}
\end{aligned}
$$


we obtain by using (3.18) and (3.17)

$$
\begin{aligned}
\delta_{w} & \left(C, P_{n}\right) \\
& \geq \frac{\operatorname{liv}_{d-1}}{2 \lambda^{9 d+2}}\left(\sum_{l=1}^{m} w_{l}^{(d-1) /(d+1)}\left(\operatorname{det} q_{l}\right)^{1 /(d+1)}\left|T_{l}^{\prime}\right|\right)^{(d+1) /(d-1)} \frac{1}{\left(n_{1}+\ldots+n_{m}\right)^{2 /(d-1)}} \\
& \geq \frac{\operatorname{liv}_{d-1}}{2 \lambda^{9 d+2}}\left(\sum_{l=1}^{m} w_{l}^{(d-1) /(d+1)}\left(\operatorname{det} q_{l}\right)^{1 /(d+1)}\left|T_{l}^{\prime}\right|\right)^{(d+1) /(d-1)} \frac{1}{n^{2 /(d-1)}} \\
& \geq \frac{\operatorname{ldiv}_{d-1}}{2 \lambda^{9 d+2+(d+1) /(d-1)}}\left(\int_{\operatorname{bd} C} w(x)^{(d-1) /(d+1)} \kappa_{C}(x)^{1 /(d+1)} d \sigma(x)\right)^{(d+1) /(d-1)} \frac{1}{n^{2 /(d-1)}}
\end{aligned}
$$

for all $n$ sufficiently large. Since $\delta_{w}\left(C, P_{n}\right)=\delta_{w}\left(C, \mathcal{P}_{(n)}\right)$, the proof is complete.

3.5 Next, we need an estimate from above for $\delta_{w}\left(C, \mathcal{P}_{(n)}\right)$.

Proposition 4 For all $n$ sufficiently large,

$\delta_{w}\left(C, \mathcal{P}_{(n)}\right) \leq \frac{\lambda^{13 d}}{2} \operatorname{ldiv}_{d-1}\left(\int_{\mathrm{bd} C} w(x)^{(d-1) /(d+1)} \kappa_{C}(x)^{1 /(d+1)} d \sigma(x)\right)^{(d+1) /(d-1)} \frac{1}{n^{2 /(d-1)}}$.

Proof. To obtain this estimate we construct a polytope $P$ with $\leq n$ facets in the following way: For $l=1, \ldots, m$, choose polyhedra with $n_{l}$ facets such that the infimum in $v_{\left(n_{l}\right)}\left(T_{l}^{\prime} ; f_{l}\right)$ is attained and define $P$ as the intersection of these polyhedra. Then, $P$ has at most $n_{1}+\ldots+n_{m}$ facets and by (3.16)

$$
\begin{aligned}
\delta_{w}(C, P) & \leq \lambda \sum_{l=1}^{m} w_{l}\left(\text { volume of the subset of } P \triangle C \text { which lies in } C_{l}\right) \\
& \leq \lambda^{2} \sum_{l=1}^{m} w_{l} v_{\left(n_{l}\right)}\left(T_{l}^{\prime} ; f_{l}\right)
\end{aligned}
$$

for $n_{1}, \ldots, n_{m}$ sufficiently large. Define $n_{l}$ as the largest integer such that

$$
n_{l} \leq \frac{w_{l}^{(d-1) /(d+1)}\left(\operatorname{det} q_{l}\right)^{1 /(d+1)}\left|T_{l}^{\prime}\right|}{\sum_{j=1}^{m} w_{j}^{(d-1) /(d+1)}\left(\operatorname{det} q_{j}\right)^{1 /(d+1)}\left|T_{j}^{\prime}\right|} n
$$

for $l=1, \ldots, m$. Then,

$$
n_{1}+\ldots+n_{m} \leq n
$$

and

$$
\frac{1}{\lambda} \frac{w_{l}^{(d-1) /(d+1)}\left(\operatorname{det} q_{l}\right)^{1 /(d+1)}\left|T_{l}^{\prime}\right|}{\sum_{j=1}^{m} w_{j}^{(d-1) /(d+1)}\left(\operatorname{det} q_{j}\right)^{1 /(d+1)}\left|T_{j}^{\prime}\right|} n \leq n_{l}
$$


for $n$ sufficiently large. We have by (3.20), Proposition 1, (3.21) and (3.17)

$$
\begin{aligned}
& \delta_{w}\left(C, \mathcal{P}_{(n)}\right) \\
& \leq \lambda^{2} \sum_{l=1}^{m} w_{l} v_{\left(n_{l}\right)}\left(T_{l}^{\prime} ; f_{l}\right) \\
& \leq \frac{\lambda^{9 d+2}}{2} \operatorname{ldiv}_{d-1} \sum_{l=1}^{m} w_{l}\left(\operatorname{det} q_{l}\right)^{1 /(d-1)}\left|T_{l}^{\prime}\right|^{(d+1) /(d-1)} \frac{1}{n_{l}^{2 /(d-1)}} \\
& \leq \frac{\lambda^{9 d+2+2 /(d-1)}}{2} \operatorname{ldiv}_{d-1}\left(\sum_{j=1}^{m} w_{j}^{(d-1) /(d+1)}\left(\operatorname{det} q_{j}\right)^{1 /(d+1)}\left|T_{j}^{\prime}\right|\right)^{2 /(d-1)} \\
& \times \sum_{l=1}^{m}\left(w_{l}^{1-2 /(d+1)}\left(\operatorname{det} q_{l}\right)^{1 /(d-1)-2 /((d-1)(d+1))}\left|T_{l}^{\prime}\right|^{(d+1) /(d-1)-2 /(d-1)}\right) \frac{1}{n^{2 /(d-1)}} \\
& \leq \frac{\lambda^{9 d+2+2 /(d-1)}}{2} \operatorname{ldiv}_{d-1}\left(\sum_{l=1}^{m} w_{l}^{(d-1) /(d+1)}\left(\operatorname{det} q_{l}\right)^{1 /(d+1)}\left|T_{l}^{\prime}\right|\right)^{(d+1) /(d-1)} \frac{1}{n^{2 /(d-1)}} \\
& \leq \frac{\lambda^{13 d}}{2} \operatorname{ldiv}_{d-1}\left(\int_{\mathrm{bd} C} w(x)^{(d-1) /(d+1)} \kappa_{C}(x)^{1 /(d+1)} d \sigma(x)\right)^{(d+1) /(d-1)} \frac{1}{n^{2 /(d-1)}}
\end{aligned}
$$

for $n$ sufficiently large. This concludes the proof of Proposition 4 .

3.5 Since $\lambda>1$ was arbitrary, (1.8) follows from Propositions 3 and 4 .

\section{Acknowledgements}

For several helpful discussions I would like to thank Prof. P.M. Gruber, Matthias Reitzner and Károly Böröczky, Jr.

\section{References}

[1] F. Aurenhammer, Power diagrams: properties, algorithms and applications, SIAM J. Comput. 16 (1987), 78-96.

[2] C. Bandle, Isoperimetric inequalities and applications, Pitman, Boston, 1980.

[3] K. Böröczky, Jr. and M. Ludwig, Approximation of convex bodies and a momentum lemma for power diagrams, Monatsh. Math. 127 (1999), 101110 .

[4] L. Fejes Tóth, Lagerungen in der Ebene, auf der Kugel und im Raum, 2nd ed., Springer, Berlin, 1972. 
[5] S. Glasauer and P.M. Gruber, Asymptotic estimates for best and stepwise approximation of convex bodies III, Forum Math. 9 (1997), 383-404.

[6] P.M. Gruber, Volume approximation of convex bodies by inscribed polytopes, Math. Ann. 281 (1988), 229-245.

[7] P.M. Gruber, Volume approximation of convex bodies by circumscribed polytopes, Applied geometry and discrete mathematics, DIMACS Ser. Discrete Math. Theoret. Comput. Sci., 4, Amer. Math. Soc., Providence, RI, 1991, 309-317.

[8] P.M. Gruber, Aspects of approximation of convex bodies, Handbook of Convex Geometry A (P.M. Gruber and J. Wills, eds.), North-Holland, Amsterdam, 1993, 319-345.

[9] P.M. Gruber, Asymptotic estimates for best and stepwise approximation of convex bodies II, Forum Math. 5 (1993), 521-538.

[10] P.M. Gruber and P. Kenderov, Approximation of convex bodies by polytopes, Rend. Circ. Mat. Palermo 31 (1982), 195-225.

[11] D. McClure and R. Vitale, Polygonal approximation of plane convex bodies, J. Math. Anal. Appl. 51 (1975), 326-358.

[12] R. Schneider, Convex bodies: the Brunn-Minkowski theory, Cambridge Univ. Press, Cambridge, 1993.

Monika Ludwig

Abteilung für Analysis

Technische Universität Wien

Wiedner Hauptstraße 8-10/1142

A 1040 Vienna 